\title{
Use of Regulatory T Cells as a Cellular Therapy for Transplant Rejection/Autoimmunity
}

\author{
Nick D Jones ${ }^{1 *}$ and Renad Alhamawi ${ }^{1,2}$ \\ ${ }^{1}$ Institute of Immunology and Immunotherapy, University of Birmingham, UK. B15 2TT \\ ${ }^{2}$ Medical laboratory Technology department, Taibah University, KSA
}

*Corresponding author: Nick D Jones, Institute of Immunology and Immunotherapy, University of Birmingham, B15 2TT, UK.

To Cite This Article: Nick D Jones. Use of Regulatory T Cells as a Cellular Therapy for Transplant Rejection/Autoimmunity. Am J Biomed Sci \& Res. 2019 - 5(2). AJBSR.MS.ID.000882. DOI: 10.34297/AJBSR.2019.05.000882

Received: 制August 14, 2019; Published: 制 September 10, 2019

\author{
Keywords: Antigen specific Treg; Autoimmunity; GVHD; Treg; Transplantation
}

Abbreviations: BAR: B cell Antibody Receptor; CAR: Chimeric Antigen Receptor; Foxp3: forkhead box protein 3; GVHD: Graft Versus Host Disease; HSC: Haematopoietic Stem Cell; TCR: T Cell Receptor; Treg: Regulatory T Cell

\section{Introduction}

Foxp3+ regulatory $\mathrm{T}$ cells (Treg) are a crucial sub-population of $\mathrm{T}$ cells in the control of immunity and the maintenance of immunological tolerance aiding the prevention of autoimmunity. As such Treg have been proposed to be a candidate cell that could be used as cellular therapy to suppress unwanted immune responses. Indeed, there is an expanding literature on the potential use of Treg to induce or re-establish tolerance in both autoimmune diseases such as type I diabetes, arthritis and chronic inflammatory diseases as well as in preventing allograft rejection and graft versus host disease (GVHD). Furthermore, the finding that antigen specific Treg are superior to polyclonal Treg in a range of disease settings has raised the possibility that Treg based therapies may be refined and improved by genetic manipulation.

The first description of Treg was by Sakaguchi and his colleagues in 1995 , where they showed the depletion of a $\mathrm{CD} 4^{+} \mathrm{CD} 25^{+} \mathrm{T}$ cell subset led to the development of autoimmune disease e.g. gastritis, thyroiditis, adrenalitis and insulitis [1]. However, CD25 is expressed by activated lymphocytes so it was the discovery that Treg express the master transcription, forkhead box protein 3 (Foxp3) that defined Treg as being a separate $\mathrm{T}$ cell lineage from conventional $\mathrm{T}$ cells [2,3]. In addition, mice that lack Foxp3 expression (scurfy mice) develop $\mathrm{T}$ cell dependent autoimmune diseases [4]. Importantly, a spontaneous inflammatory disease called Immune dysregulation polyendocrinopathy enteropathy $\mathrm{X}$ linked syndrome (IPEX) is the result of a mutation in the foxp3 gene which links Treg and foxp3 to the development of autoimmunity in humans as well as in mice [4]. The mechanisms employed by Treg to suppress immunity can be mediated by cell contact (e.g. via CTLA-4) or may be cell contactindependent (e.g. IL-10 and TGF $\beta$ ) [5].
Treg can be isolated from a number of different sources including peripheral blood, umbilical cord blood and discarded paediatric thymuses, however, the most common source for Treg thus far used in clinical trials is peripheral blood. In order to take advantage of the suppressive capacity of such cells and due to the low frequency of Treg in peripheral blood, ex-vivo expansion of the cells using non-specific stimulation with anti-CD3 $\backslash$ CD28 in the presence of a high concentration of IL-2 is generally required. This type of expansion protocol has proved to be able to successfully generate sufficient numbers of Treg to be infused in clinical trials [6].

Indeed, Treg ex-vivo expanded in this way have been introduced as an immunotherapeutic agent in autoimmune diseases such as type 1 diabetes $[7,8]$. It is worth noting that in all studies to date the administration of ex-vivo expanded Treg to patients has not resulted in any serious complications or side-effects. A recent study reported that the infused Tregs can be detected in the circulation for at least 3 months post infusion and that such cells largely retain their original Treg phenotype [9]. Interestingly, it has been shown that culturing Treg together with certain cytokine combinations during ex-vivo expansion may result in Treg expressing chemokine receptors that may facilitate Treg migration to sites of inflammation and/or peripheral tissues [10].

Ex-vivo expanded Treg have now been infused into patients in a number of different disease settings. For example, such Treg have been transferred to patients at risk of acute and chronic graft versus host disease (GVHD); a condition that can occur following allogeneic HSC transplantation due to alloreactive T lymphocytes present in the donor HSC product. These studies demonstrated 
that it is feasible and safe to inject Treg into patients receiving HSC transplantation and that their infusion correlated with the suppression of GVHD and enhanced immune recovery [11,12]. Ex-vivo expanded Tregs have also been administered to liver and kidney transplant patients $[13,14]$. Indeed, the ONE study intensively investigated the safety and feasibility of injecting ex vivo expanded Treg into kidney and liver transplant patients and has reported no side-effects associated with the infused Treg $[13,15]$. The contribution, if any, of the administered Treg to the suppression of rejection has yet to be confirmed as both sets of patients received conventional immunosuppressive agents in addition to the Treg.

The Tregs that have been given to patients are largely the result of polyclonal expansion such that only a small percentage of the Treg are likely to recognise the target disease associated antigen/alloantigen. However, advances in gene-engineering may have opened an avenue to re-direct Treg specificity toward a particular antigen thereby potentially increasing the suppressive capacity of the Treg whilst lowering the possibility of off-target immunosuppression [16]. Indeed, $\mathrm{T}$ cell receptor (TCR) or chimeric antigen receptor (CAR) gene engineering of conventional $\mathrm{T}$ cells has been widely trailed to treat solid and haematological tumours and such clinical trials have shown encouraging and often remarkable results [17-19]. The successful outcomes from re-directing conventional $\mathrm{T}$ cells toward tumour antigens through either TCR or CAR gene engineering raises the question of whether similar approaches could be applied to regulatory T cells in order to suppress autoimmunity and allograft rejection.

A recent study highlighted the potential of CAR-Treg showing that lung epithelium-reactive CAR-Tregs demonstrated an enhanced ability to suppress allergic airway inflammation in a mouse model compared to their polyclonal counterparts [20]. Moreover, directing Treg specificity to ta issue-specific antigen or disease related antigen revealed a robust suppression of Type I diabetes and rheumatoid arthritis [21,22]. In terms of transplantation, several recent studies have shown that HLA-A2-specific CAR Tregs displayed an enhanced ability to suppress HLA-A2+ skin allograft rejection and xenogeneic GVHD in humanised mouse models of transplantation [23-25]. Another promising approach in the field is the use of an engineered $\mathrm{B}$ cell antibody receptor (BAR) in conventional $\mathrm{T}$ cells or Treg to ameliorate B cell mediated autoimmunity and alloimmunity [26]. Recently, BAR transduced Treg have been shown to potently prevent the secretion of the neutralising antibodies that develop against the therapeutic factor VIII in haemophilia [27]. This raises the question whether using BAR Treg would be advantageous to suppress pathogenic antibodies in autoimmunity and transplantation.

Taken together there is now convincing evidence that Treg can be isolated and expanded from patients under cGMP conditions to enable their re-infusion as part of a therapeutic strategy. Phase 1 clinical trials have shown that these cells are able to survive for prolonged periods of time post infusion and that the generation of location-specific or antigen-specific Treg is likely to increase the potency of such cells. However, it remains to be seen whether Treg can be used as a cellular therapy for autoimmune disease and allograft rejection/GVHD in isolation or perhaps more likely will be used as part of an adjunctive strategy combined with more traditional immunosuppressive agents. The other important point is whether Tregs when confronted with certain inflammatory stimuli could lose their suppressive phenotype and convert to $\mathrm{T}$ helper subsets such as IFN $\gamma$ or IL-17 secreting CD4+ T cells that may exacerbate rather than suppress immune-mediated disease [28]. Addressing these potential confounding factors will be a necessary next step in the potential implementation of Treg as a cellular therapy for autoimmunity, transplant rejection and GVHD.

\section{References}

1. Sakaguchi S, Sakaguchi N, Asano M, Itoh M, Toda M (1995) Immunologic self-tolerance maintained by activated $\mathrm{T}$ cells expressing IL-2 receptor alpha-chains (CD25). Breakdown of a single mechanism of self-tolerance causes various autoimmune diseases. J Immunol 155(3): 1151-1164.

2. Hori S, Nomura T, Sakaguchi S (2003) Control of regulatory T cell development by the transcription factor Foxp3. Science 299(5609): 10571061.

3. Fontenot JD, Gavin MA, Rudensky AY (2003) Foxp3 programs the development and function of CD4+CD25+ regulatory T cells. Nat Immunol 4(4): 330-336.

4. Sakaguchi S (2005) Naturally arising Foxp3-expressing CD25+ CD4+ regulatory $\mathrm{T}$ cells in immunological tolerance to self and non-self. Nat Immunol 6(4): 345-352.

5. Sakaguchi S, Wing K, Onishi Y, Prieto Martin P, Yamaguchi T (2009) Regulatory T cells: how do they suppress immune responses? International immunology 21(10): 1105-1111.

6. Vaikunthanathan T, Safinia N, Boardman D, Lechler R, Lombardi G (2017) Regulatory T cells: tolerance induction in solid organ transplantation. Clinical \& Experimental Immunology 189(2): 197-210.

7. Marek Trzonkowska N, Myśliwiec M, Dobyszuk A, Grabowska M, Techmańska I, et al. (2012) Administration of CD4+ CD25highCD127- regulatory $\mathrm{T}$ cells preserves $\beta$-cell function in type 1 diabetes in children. Diabetes care 35(9): 1817-1820.

8. Bluestone JA, Buckner JH, Fitch M, Gitelman SE, Gupta S, et al. (2015) Type 1 diabetes immunotherapy using polyclonal regulatory T cells. Science translational medicine 7(315): 315ra189.

9. Chandran S, Tang Q, Sarwal M, Laszik ZG, Putnam AL, et al. (2017) Polyclonal Regulatory $\mathrm{T}$ Cell Therapy for Control of Inflammation in Kidney Transplants. Am J Transplant 17(11): 2945-2954.

10. Hoeppli RE, MacDonald KN, Leclair P, Fung VCW, Mojibian M, et al. (2019) Tailoring the homing capacity of human Tregs for directed migration to sites of Th1-inflammation or intestinal regions. Am J Transplant 19(1): 62-76.

11. Tang Q Bluestone JA (2013) Regulatory T-cell therapy in transplantation: moving to the clinic. Cold Spring Harb Perspect Med 3(11).

12. Di Ianni M, Falzetti F, Carotti A, Terenzi A, Castellino F, et al. (2011) Tregs prevent GVHD and promote immune reconstitution in HLA-haploidentical transplantation. Blood 117(14): 3921-3928.

13. Juvet SC, Whatcott AG, Bushell AR, Wood KJ (2014) Harnessing regulatory $\mathrm{T}$ cells for clinical use in transplantation: the end of the beginning. Am J Transplant 14(4): 750-763.

14. Edozie FC, Nova-Lamperti EA, Povoleri GA, Scottà C, John S, et al. (2014) Regulatory T-cell therapy in the induction of transplant tolerance: the issue of subpopulations. Transplantation 98(4): 370-379.

15. Romano M, Fanelli G, Albany CJ, Giganti G, Lombardi G (2019) Past, Present, and Future of Regulatory T Cell Therapy in Transplantation and Autoimmunity. Front Immunol 10:43. 
16. Jethwa H, Adami AA, Maher J (2014) Use of gene-modified regulatory T-cells to control autoimmune and alloimmune pathology: is now the right time? Clin Immunol 150(1): 51-63.

17. Zhao Y, Zheng Z, Robbins PF, Khong HT, Rosenberg SA, et al. (2005) Primary human lymphocytes transduced with NY-ESO-1 antigen-specific TCR genes recognize and kill diverse human tumor cell lines. J Immunol 174(7): 4415-4423.

18. Zhang L, Morgan RA (2012) Genetic engineering with T cell receptors. Adv Drug Deliv Rev 64(8): 756-762.

19. Hay KA, Turtle CJ (2017) Chimeric Antigen Receptor (CAR) T Cells: Lessons Learned from Targeting of CD19 in B-Cell Malignancies. Drugs 77(3): 237-245.

20. Skuljec J, Chmielewski M, Happle C, Habener A, Busse M, et al. (2017) Chimeric antigen receptor-redirected regulatory $\mathrm{T}$ cells suppress experimental allergic airway inflammation, a model of asthma. Front Immunol 8: 1125.

21. Hull CM, Nickolay LE, Estorninho M, Richardson MW, Riley JL, et al. (2017) Generation of human islet-specific regulatory T cells by TCR gene transfer. Journal of autoimmunity 79: 63-73.

22. Wright GP, Notley CA, Xue S-A, Bendle GM, Holler A, et al. (2009) Adoptive therapy with redirected primary regulatory $\mathrm{T}$ cells results in antigen-specific suppression of arthritis. Proc Natl Acad Sci U S A 106(45): 19078-19083.
23. Noyan F, Zimmermann K, Hardtke Wolenski M, Knoefel A, Schulde E, et al. (2017) Prevention of allograft rejection by use of regulatory $\mathrm{T}$ cells with an MHC-specific chimeric antigen receptor. Am J Transplant 17(4): 917-930.

24. MacDonald KG, Hoeppli RE, Huang Q, Gillies J, Luciani DS, et al. (2016) Alloantigen-specific regulatory $\mathrm{T}$ cells generated with a chimeric antigen receptor. J Clin Invest 126(4): 1413-1424.

25. Boardman DA, Philippeos C, Fruhwirth GO, Ibrahim MA, Hannen RF, et al. (2017) Expression of a chimeric antigen receptor specific for donor HLA class I enhances the potency of human regulatory $\mathrm{T}$ cells in preventing human skin transplant rejection. Am J Transplant 17(4): 931-943.

26. Sicard A, Levings MK, Scott DW (2018) Engineering therapeutic T cells to suppress alloimmune responses using TCRs, CARs, or BARs. Am J Transplant 18(6): 1305-1311.

27. Zhang AH, Yoon JH, Kim YC, Scott DW (2016) Targeting FVIII-specific B cells using BAR-transduced regulatory T cells. Am Soc Hematology 128: 329.

28. Hua J, Inomata T, Chen Y, Foulsham W, Stevenson W, et al. (2018) Pathological conversion of regulatory T cells is associated with loss of allotolerance. Sci Rep 8(1): 7059. 